\title{
On-line prediction of the fatty acid content of beef meat by near infrared reflectance spectroscopy
}

N Prieto ${ }^{1}$, D W Ross ${ }^{1}$, E A Navajas ${ }^{1}$, G Nute ${ }^{2}$, R I Richardson ${ }^{2}$, J J Hyslop ${ }^{1}$, G Simm $^{1}$, R Roehe ${ }^{1}$

${ }^{1}$ Scottish Agricultural College, Edinburgh, United Kingdom, ${ }^{2}$ University of Bristol, Bristol, United Kingdom

Email: etcnpb00@estudiantes.unileon.es

Introduction The amount and fatty acid (FA) composition of beef intramuscular fat (IMF) are key factors that influence technological and sensory quality, especially shelf-life (lipid and pigment oxidation) and flavour. Furthermore, consumers are interested in the fat composition of meat, as nutritional guidelines are recommending a lower saturated FA (SFA) intake due to its association with cardiovascular diseases. The amount and composition of ruminant IMF, which depends on factors such as the genetic origin of the animals, feeding regime, age or live weight, influences the final quality of the product, which also explains the increasing interest in defining the FA profile of meat. However, quantitative chemical techniques for the determination of FA involve extraction of total lipids and determination of FA methyl esters by gas chromatography, so that this procedure is costly, time-consuming and generates hazardous waste. The use of near infrared reflectance spectroscopy (NIR) is increasing in food analysis because it offers several advantages over conventional methods, giving fast, non-destructive, clean and cost effective measurements. Therefore, the aim of this study was to test the on-line estimation of the concentration of major individual FA (C16:0, C18:0 and C18:1) and main groups of FA (SFA, monounsaturated FA (MUFA) and polyunsaturated FA (PUFA)) of beef IMF using NIR, by direct application of a fibreoptic probe to the $M$. longissimus thoracis with no prior sample treatment.

Materials and methods Cross-bred steers and heifers $(n=194$; sired by either Aberdeen Angus or Limousin bulls $)$ were used in this three-year study. The animals were slaughtered in 11 batches from autumn 2006 until spring 2008 at an average carcass weight and age at slaughter of $335(\mathrm{SD}=30.3) \mathrm{kg}$ and $584(\mathrm{SD}=26.2)$ days, respectively. NIR measurements were taken from the left side carcass $M$. longissimus thoracis (13th rib region) at 48 hours post mortem (pm), by means a NIR spectrophotometer (Qualityspec Pro, ASD Inc., Boulders, Colorado) provided with a sampling fibre-optic probe. Meat samples were scanned at every $1 \mathrm{~nm}$ over the NIR spectral range (350 to $1,800 \mathrm{~nm}$ ). The absorbance data were subjected to multiplicative scatter correction and/or derivatives. Full cross-validation was applied and the best equation was selected on the basis of minimising the standard error of cross-validation $\left(\mathrm{SE}_{\mathrm{CV}}\right)$. One inch thick steak from the M. longissimus lumborum from the cranial end was vacuum packed at 48 hours pm and frozen immediately until the fatty acid composition was determined. Fatty acid analysis was carried out by direct saponification as described in detail by Teye et al. (2006). Samples were hydrolysed with $2 \mathrm{M} \mathrm{KOH}$ in water:methanol $(1: 1)$ and the fatty acids extracted into petroleum spirit, methylated using diazomethane and analysed by gas liquid chromatography.

Results From the 194 beef samples, the highest correlations between NIR absorbance data transformed to second-order derivative spectra and major individual FA (C16:0, C18:0 and C18:1) were located at wavelengths of 950, 1100-1400 and 1650-1780 nm, which correspond to absorption by C-H bonds related to long C-H chains of fatty acids (Figure 1). Hence, it is possible that information related to the FA composition of the beef samples could be explained using absorbance data. In order to validate this hypothesis, prediction equations were developed. The prediction results using the fibre-optic probe showed partial least squares correlations $\left(\mathrm{SE}_{\mathrm{CV}}, \mathrm{g} .100 \mathrm{~g}^{-1}\right.$ muscle) for $\mathrm{C} 16: 0, \mathrm{C} 18: 0, \mathrm{C} 18: 1$, SFA, MUFA and PUFA of $0.784(0.22), 0.684(0.10), 0.785(0.33), 0.742(0.35), 0.797(0.37)$ and $0.488(0.03)$, respectively.

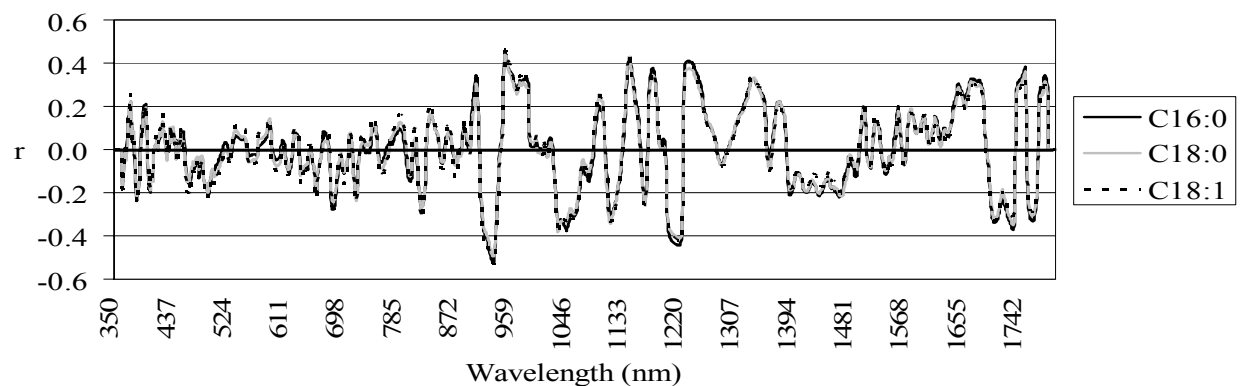

Figure 1 Correlation coefficients (r) between individual FA content and the absorbance data of the second-order derivative spectra of beef samples

Conclusions The moderate to high correlations between NIR measurements and FA content indicate that NIR measurements recorded in the abattoir could be used for the prediction of FA content of beef meat. Saturated and monounsaturated FA were more accurately predicted than polyunsaturated FA. In summary, the results of this study suggest that NIR spectroscopy could be used on-line as a fast and relatively inexpensive estimation of FA in beef, which may be exploited by the abattoir for a value-based marketing system or by breeding organisations for genetic improvement of meat quality.

Acknowledgements We are grateful to the Scottish Government for funding the research and Scotbeef, QMS, BCF and Signet for their substantial support.

\section{References}

Teye, G.A., Sheard, P.R., Whittington, F.M., Nute, G.R., Stewart, A. and Wood, J.D. 2006. Meat Science 73, $157-165$. 